\title{
Some Further Results on Weighted Sharing of Values for Meromorphic Functions Concerning a Result of Terglane
}

\author{
XiaO-Min Li \\ Department of Mathematics, Ocean University of China, Qingdao, Shandong \\ 266071, China \\ e-mail : xmli01267@gmail.com

\section{Hong-Xun Yi} \\ Department of Mathematics, Shandong University, Jinan, Shandong 250100, China \\ e-mail : hxyi@sdu.edu.cn
}

ABSTRACT. In this paper, we deal with the problem of meromorphic functions that have three weighted sharing values, and obtain some uniqueness theorems which improve those given by N. Terglane, Hong-Xun Yi \& Xiao-Min Li, and others. Some examples are provided to show that the results in this paper are best possible.

\section{Introduction and main results}

In this paper, by meromorphic functions we will always mean meromorphic functions in the complex plane. We adopt the standard notations in the Nevanlinna theory of meromorphic functions as explained in [3]. It will be convenient to let $E$ denote any set of positive real numbers of finite linear measure, not necessarily the same at each occurrence. For any nonconstant meromorphic function $h(z)$, we denote by $S(r, h)$ any quantity satisfying $S(r, h)=o(T(r, h)) \quad(r \rightarrow \infty, r \notin E)$.

Let $f(z)$ and $g(z)$ be two nonconstant meromorphic functions, and let $a \in$ $C \cup\{\infty\}$, where $C \cup\{\infty\}$ denotes the extended complex plane. We denote by $\bar{N}_{0}(r, a, f, g)$ the counting function of the common zeros of $f(z)-a$ and $g(z)-a$, and each point is counted only once, where $f(z)-\infty$ means $1 / f(z)$ (see [10]). We say that $f$ and $g$ share the value $a \mathrm{CM}$, provided that $f$ and $g$ have the same $a$-points with the same multiplicities. Similarly, we say that $f$ and $g$ share the value $a$ IM, provided that $f$ and $g$ have the same $a$-points ignoring multiplicities (see [12]). Throughout this paper, we denote by $\bar{N}_{(k, l)}(r, a)$ the reduced counting function of those points in $\bar{N}(r, 1 /(f-a))$, such that $a$ is taken by $f$ with multiplicity $k$, and such that $a$ is taken by $g$ with multiplicity $l$. In this paper, we also need the following

Received April 20, 2007, and, in revised form, August 3, 2007.

2000 Mathematics Subject Classification: 30D30, 30D35.

Key words and phrases: meromorphic functions, weighted sharing values, uniqueness theorems.

Project supported by the NSFC (No. A0324617), the RFDP (No. 20060422049) and the NSFC(No. 10771121). 
definition.

Definition 1.1 ([1, Definition 1]). Let $p$ be a positive integer and $a \in C \cup\{\infty\}$. Then by $N_{p)}\left(r, \frac{1}{f-a}\right)$ we denote the counting function of those zeros of $f-a$ (counted with proper multiplicities) whose multiplicities are not greater than $p$, by $\bar{N}_{p)}\left(r, \frac{1}{f-a}\right)$ we denote the corresponding reduced counting function (ignoring multiplicities). By $N_{(p}\left(r, \frac{1}{f-a}\right)$ we denote the counting function of those zeros of $f-a$ (counted with proper multiplicities) whose multiplicities are not less than $p$, by $\bar{N}_{(p}\left(r, \frac{1}{f-a}\right)$ we denote the corresponding reduced counting function (ignoring multiplicities).

Let $a_{1}, a_{2}, a_{3}$ and $a_{4}$ be four distinct elements in $C \cup\{\infty\}$. If $a_{j} \neq \infty(j=$ $1,2,3,4)$, we define their cross ratio to be

$$
\left(a_{1}, a_{2}, a_{3}, a_{4}\right)=\frac{a_{1}-a_{3}}{a_{2}-a_{3}}: \frac{a_{1}-a_{4}}{a_{2}-a_{4}} .
$$

If $a_{k}=\infty(1 \leq k \leq 4)$, define their cross ratio to be

$$
\left(a_{1}, a_{2}, a_{3}, a_{4}\right)=\lim _{a_{k} \rightarrow \infty} \frac{\left(a_{1}-a_{3}\right)\left(a_{2}-a_{4}\right)}{\left(a_{2}-a_{3}\right)\left(a_{1}-a_{4}\right)} .
$$

If $A \in C$ and $A \neq 0,1$, then $(A, 1,0, \infty)=A$. Throughout this paper, let $a, b, c, d$ be four distinct elements in $C \cup\{\infty\}$, and let

$$
L(w)=\frac{(w-c)(b-d)}{(w-d)(b-c)} .
$$

It is obvious that

$$
L(a)=(a, b, c, d) .
$$

In 1989, N. Terglane proved the following result.

Theorem A ([11, P. 61, Theorem 3.14]). Let $f$ and $g$ be nonconstant meromorphic functions and let $a, b, c$ and $d$ be four distinct complex numbers such that $(a, b, c, d) \in$ $\left\{-1,2, \frac{1}{2}\right\}$. If $f$ and $g$ share $b, c, d C M$, and if

$$
\bar{N}_{0}(r, a, f, g) \neq S(r, f)
$$

then $f$ is a Möbius transformation of $g$.

Now it is natural to ask the following two questions.

Question 1.1 ([4, Question 1]). What can be said if we get rid of the condition " $(a, b, c, d) \in\left\{-1,2, \frac{1}{2}\right\}$ " in Theorem A ?

Question $1.2([5])$. Is it really possible to relax in any way the nature of sharing any one of $a, b$ and $c$ in Theorem A ? 
Let $f$ and $g$ share 0,1 and $\infty$ IM, next we denote by $N_{0}(r)$ the counting function of the zeros of $f-g$ not containing the zeros of $f, 1 / f$ and $f-1$.

In 2003, the first question is answered by X. M. Li and H. X. Yi in the following theorem.

Theorem $\mathbf{B}([4$, Theorem 1$])$. Let $f$ and $g$ be two distinct nonconstant meromorphic functions sharing 0,1 and $\infty C M$, and let $a(\neq 0,1)$ be a finite complex number such that (1.3) holds, then $f$ is a Möbius transformation of $g$, apart from the following three exceptional cases:

(i) $f \equiv \frac{e^{s_{1} \gamma}-1}{e^{\left(k_{1}+1\right) \gamma}-1}, \quad g \equiv \frac{e^{-s_{1} \gamma}-1}{e^{-\left(k_{1}+1\right) \gamma}-1}$, with $1 \leq s_{1} \leq k_{1}$ and $a=\frac{s_{1}}{k_{1}+1}$,

(ii) $f \equiv \frac{e^{\left(k_{1}+1\right) \gamma}-1}{e^{\left(k_{1}+1-s_{1}\right) \gamma}-1}, g \equiv \frac{e^{-\left(k_{1}+1\right) \gamma}-1}{e^{-\left(k_{1}+1-s_{1}\right) \gamma}-1}$, with $1 \leq s_{1} \leq k_{1}$ and $a=\frac{k_{1}+1}{k_{1}+1-s_{1}}$,

(iii) $f \equiv \frac{e^{s_{1} \gamma}-1}{e^{-\left(k_{1}+1-s_{1}\right) \gamma}-1}, \quad g \equiv \frac{e^{-s_{1} \gamma}-1}{e^{\left(k_{1}+1-s_{1}\right) \gamma}-1}$, with $1 \leq s_{1} \leq k_{1}$ and $a=\frac{s_{1}}{s_{1}-k_{1}-1}$,

where $k_{1}(\geq 2)$ and $s_{1}$ are positive integers such that $s_{1}$ and $k_{1}+1$ are relatively prime, and $\gamma$ is a nonconstant entire function. Furthermore, the following equality holds:

$$
\bar{N}_{(1,1)}(r, a)=N_{0}(r)+S(r, f)=\frac{1}{k_{1}} T(r, f)+S(r, f) .
$$

In this paper, we will deal with Question 1.2. To this end we employ the idea of weighted sharing of values which measures how close a shared value is to being shared IM or to being shared CM. The notion is explained in the following definition.

Definition 1.2([6, Definition 4]). Let $k$ be a nonnegative integer or infinity. For any $a \in C \cup\{\infty\}$, we denote by $E_{k}(a, f)$ the set of all $a$-points of $f$, where an $a$-point of multiplicity $m$ is counted $m$ times if $m \leq k$, and $k+1$ times if $m>k$. If $E_{k}(a, f)=E_{k}(a, g)$, we say that $f, g$ share the value $a$ with weight $k$.

Remark 1.1. Definition 1.2 implies that if $f, g$ share a value $a$ with weight $k$, then $z_{0}$ is a zero of $f-a$ with multiplicity $m(\leq k)$ if and only if it is a zero of $g-a$ with multiplicity $m(\leq k)$, and $z_{0}$ is a zero of $f-a$ with multiplicity $m(>k)$, if and only if it is a zero of $g-a$ with multiplicity $n(>k)$, where $m$ is not necessarily equal to $n$. Throughout this paper, we write $f, g$ share $(a, k)$ to mean that $f, g$ share the value $a$ with weight $k$. Clearly, if $f, g$ share $(a, k)$, then $f, g$ share $(a, p)$ for all integer $p, 0 \leq p<k$. Also we note that $f, g$ share a value $a$ IM or CM if and only if $f, g$ share $(a, 0)$ or $(a, \infty)$, respectively.

Using the idea of weighted sharing, we will establish the following theorem, which improves Theorem B and deals with Question 1.2.

Theorem 1.1. Let $f$ and $g$ be two distinct nonconstant meromorphic functions sharing $\left(0, k_{1}\right),\left(1, k_{2}\right)$ and $\left(\infty, k_{3}\right)$, where $k_{1}, k_{2}$ and $k_{3}$ are three positive integers satisfying

$$
k_{1} k_{2} k_{3}>k_{1}+k_{2}+k_{3}+2
$$


and let $a(\neq 0,1)$ be a finite complex number such that (1.3) holds. If $f$ is a Möbius transformation of $g$, then

$$
\bar{N}_{0}(r, a, f, g)=T(r, f)+S(r, f)
$$

and there exists a nonconstant entire function $\gamma$ such that $f$ and $g$ are given by one of the following three expressions:

(a) $f=e^{\gamma}$ and $g=e^{-\gamma}$, where $a=-1$,

(b) $f=e^{\gamma}+1$ and $g=e^{-\gamma}+1$, where $a=2$,

(c) $f=\frac{1}{e^{\gamma}+1}$ and $g=\frac{1}{e^{-\gamma}+1}$, where $a=1 / 2$.

If $f$ is not any Möbius transformation of $g$, then there exist two positive integers $k_{1}(\geq 2)$ and $s_{1}$ that are relatively prime, and there exists a nonconstant entire function $\gamma$, such that (1.4) holds, and such that $f$ and $g$ are given by one of the three expressions (i), (ii) and (iii) in Theorem B.

Example 1.1. Let $f \equiv \frac{e^{z^{2}}-1}{e^{z}-1}, \quad g \equiv \frac{e^{-z^{2}}-1}{e^{-z}-1}$ and let $a$ be a complex number satisfying $a \neq 0,1, \infty$. Then $f$ and $g$ share $(0,1),(1,2)$ and $(\infty, 6)$, furthermore, $\bar{N}_{0}(r, a, f, g)=S(r, f)$, we can verify that $f$ is not any Möbius transformation of $g$, and the three cases (i), (ii) and (iii) in Theorem B can not occur. This example shows that the condition (1.3) in Theorem 1.1 is best possible.

From Theorem 1.1 we deduce the following two corollaries.

Corollary 1.1. Let $f$ and $g$ be two nonconstant meromorphic functions sharing $\left(0, k_{1}\right),\left(1, k_{2}\right)$ and $\left(\infty, k_{3}\right)$, where $k_{1}, k_{2}$ and $k_{3}$ are three positive integers satisfying (1.5), and let $a(\neq 0,1)$ be a finite complex number such that (1.3) holds. If for any positive integer $n, \bar{N}_{(1,1)}(r, a) \neq \frac{1}{n} T(r, f)+S(r, f)$, then $f \equiv g$.

Corollary 1.2. Let $f$ and $g$ be two nonconstant meromorphic functions sharing $\left(0, k_{1}\right),\left(1, k_{2}\right)$ and $\left(\infty, k_{3}\right)$, where $k_{1}, k_{2}$ and $k_{3}$ are three positive integers satisfying (1.5), and let a be a finite nonrational complex number. If (1.3) holds, then $f \equiv g$.

In 2002, H. X. Yi and X. M. Li proved the following theorem.

Theorem $\mathbf{C}([13$, Theorem 1.1]). Let $f$ and $g$ be two nonconstant meromorphic functions, and let $a_{1}, a_{2}, b, c$ and $d$ be five distinct elements in $C \cup\{\infty\}$. If $f$ and $g$ share $b, c$ and $d C M$, and if

$$
\begin{aligned}
& \bar{N}_{0}\left(r, a_{1}, f, g\right) \neq S(r, f), \\
& \bar{N}_{0}\left(r, a_{2}, f, g\right) \neq S(r, f)
\end{aligned}
$$

then $f \equiv g$.

In this paper, we will prove the following theorem, which improves Theorem C.

Theorem 1.2. Let $f$ and $g$ be two nonconstant meromorphic functions sharing $\left(0, k_{1}\right),\left(1, k_{2}\right)$ and $\left(\infty, k_{3}\right)$, where $k_{1}, k_{2}$ and $k_{3}$ are three positive integers satisfying 
(1.5), and let $a_{1}(\neq 0,1)$ and $a_{2}(\neq 0,1)$ be two distinct finite complex numbers. If (1.7) and (1.8) hold, then $f \equiv g$.

Example 1.2. Let $f(z)=e^{-z}, \quad g(z)=e^{z}$. Then it is verified that $f$ and $g$ share $(0,1),(1,2)$ and $(\infty, 6)$, and $\bar{N}_{0}(r,-1, f, g)=T(r, f)+S(r, f)$. Furthermore, we verify that for any $b \in C \backslash\{-1,0,1$,$\} , we have \bar{N}_{0}(r, b, f, g)=0$. However, $f \not \equiv g$. This example illustrates that we can not delete either of the condition (1.7) and (1.8) in Theorem 1.2.

In 2002, H. X. Yi and X. M. Li proved the following theorem.

Theorem $\mathbf{D}([13$, Theorem 1.2]). Let $f$ and $g$ be two distinct nonconstant meromorphic functions, and let $a, b, c$ and $d$ be four distinct elements in $C \cup\{\infty\}$. If $f$ and $g$ share $b, c$ and $d C M$, and if (1.3) holds, then $(a, b, c, d)$ is a rational number, and

$$
\begin{aligned}
& \bar{N}\left(r, \frac{1}{f-a}\right)=T(r, f)+S(r, f), \\
& \bar{N}\left(r, \frac{1}{g-a}\right)=T(r, g)+S(r, f)
\end{aligned}
$$

and

$$
\bar{N}_{0}(r, a, f, g)=\frac{1}{k_{1}} T(r, f)+S(r, f),
$$

where $k_{1}$ is a positive integer.

In this paper, we will prove the following theorem, which improves Theorem D.

Theorem 1.3. Let $f$ and $g$ be two distinct nonconstant meromorphic functions sharing $\left(0, k_{1}\right),\left(1, k_{2}\right)$ and $\left(\infty, k_{3}\right)$, where $k_{1}, k_{2}$ and $k_{3}$ are three positive integers satisfying $(1.5)$, and let $a(\neq 0,1)$ be a finite complex number such that (1.3) holds, then $a$ is a rational number, and (1.9), (1.10) and (1.11) still hold.

Example 1.3. Let $f(z)=\frac{e^{z}-1}{e^{z}+1}, \quad g(z)=\frac{e^{-z}-1}{e^{-z}+1}$. Then it is verified that $f$ and $g$ share $(0,1),(1,2)$ and $(\infty, 6)$, and $f$ is a fractional linear transformation of $g$. Moreover, $\bar{N}\left(r, \frac{1}{f+1}\right)=0, \bar{N}\left(r, \frac{1}{g+1}\right)=0$ and $\bar{N}_{0}(r,-1, f, g)=0$.

This example illustrates the condition (1.3) in Theorem 1.3 is necessary.

Example 1.4. Let $f(z)=e^{2 z}+e^{z}+1, g(z)=e^{-2 z}+e^{-z}+1$. Then it is verified that $f$ and $g$ share $(0,1),(1,2)$ and $(\infty, 6)$, and $f$ is not any fractional linear transformation of $g$. Moreover, $\bar{N}\left(r, \frac{1}{f-3 / 4}\right)=T(r, f) / 2+S(r, f), \bar{N}\left(r, \frac{1}{g-3 / 4}\right)=T(r, g) / 2+S(r, g)$ and $\bar{N}_{0}(r, 3 / 4, f, g)=0$.

This example also illustrates the condition (1.3) in Theorem 1.3 is necessary. From Theorem 1.3 we deduce the following corollary. 
Corollary 1.3. Let $f$ and $g$ be two nonconstant meromorphic functions sharing $\left(0, k_{1}\right),\left(1, k_{2}\right)$ and $\left(\infty, k_{3}\right)$, where $k_{1}, k_{2}$ and $k_{3}$ are three positive integers satisfying $(1.5)$, and let $a(\neq 0,1)$ be a finite complex number such that (1.3) holds. If

$$
\bar{N}\left(r, \frac{1}{f-a}\right) \neq T(r, f)+S(r, f),
$$

then $f \equiv g$.

In 2002, H. X. Yi and X. M. Li proved the following theorem.

Theorem $\mathbf{E}([13$, Theorem 1.3]). Let $f$ and $g$ be two distinct nonconstant meromorphic functions, and let $a, b, c$ and $d$ be four distinct elements in $C \cup\{\infty\}$. If $f$ and $g$ share $b, c$ and $d C M$, and if (1.6) holds, then $(a, b, c, d) \in\left\{-1,2, \frac{1}{2}\right\}$, and $f$ is a fractional linear transformation of $g$, and assumes one of the following relations:

(i) $L(f)=e^{\gamma}, \quad L(g)=e^{-\gamma}$, this occurs only for $(a, b, c, d)=-1$

(ii) $L(f)=e^{\gamma}+1, \quad L(g)=e^{-\gamma}+1$, this occurs only for $(a, b, c, d)=2$

(ii) $L(f)=\frac{1}{e^{\gamma}+1}, \quad L(g)=\frac{1}{e^{-\gamma}+1}$, this occurs only for $(a, b, c, d)=\frac{1}{2}$

From Theorem 1.1 we deduce the following theorem, which improves Theorem E.

Theorem 1.4. Let $f$ and $g$ be two distinct nonconstant meromorphic functions sharing $\left(0, k_{1}\right),\left(1, k_{2}\right)$ and $\left(\infty, k_{3}\right)$, where $k_{1}, k_{2}$ and $k_{3}$ are three positive integers satisfying (1.5), and let $a(\neq 0,1)$ be a finite complex number such that (1.6) holds, then $a \in\left\{-1,2, \frac{1}{2}\right\}$, and $f$ is a fractional linear transformation (Möbius transformation) of $g$, furthermore, there exists a nonconstant entire function $\gamma$ such that $f$ and $g$ are given by one of the three expressions (a), (b) and (c) in Theorem 1.1.

Example 1.5. Let $f(z)=\frac{1-e^{z}}{2}, g(z)=\frac{1-e^{-z}}{2}$. Then it is verified that $f$ and $g$ share $(0,1),(1,2)$ and $(\infty, 6)$, and $\bar{N}_{0}(r, a, f, g)=0$, where $a \in C \backslash\{0,1\}$ is an arbitrary finite complex number. Moreover, we see that $f$ is a fractional linear transformation of $g$, however, the three cases (a), (b) and (c) in Theorem 1.4 can not occur. From this example and Theorem 1.1 we see that the condition (1.6) of Theorem 1.4 is necessary.

In 2002, H. X. Yi and X. M. Li proved the following theorem.

Theorem $\mathbf{F}([13$, Theorem 1.4]). Let $f$ and $g$ be two distinct nonconstant meromorphic functions, and let $a, b, c$ and $d$ be four distinct elements in $C \cup\{\infty\}$. If $f$ and $g$ share $b, c$ and $d C M$, and if (1.3) and

$$
\bar{N}_{0}(r, a, f, g) \neq T(r, f)+S(r, f)
$$

hold, then $(a, b, c, d) \notin\left\{1,0,-1,2, \frac{1}{2}\right\}$ is a rational number, and there exist two positive integers $k_{1}(\geq 2)$ and $s_{1}$ such that $k_{1}+1$ and $s_{1}$ are relatively prime, and there exists a nonconstant entire function $\gamma$, such that (1.4) holds, and such that $f$ and $g$ are given by one of the following three cases. 
(i) $L(f) \equiv \frac{e^{s_{1} \gamma}-1}{e^{\left(k_{1}+1\right) \gamma}-1}, g \equiv \frac{e^{-s_{1} \gamma}-1}{e^{-\left(k_{1}+1\right) \gamma}-1}$, with $1 \leq s_{1} \leq k_{1}$ and $(a, b, c, d)=\frac{s_{1}}{k_{1}+1}$,

(ii) $L(f) \equiv \frac{e^{\left(k_{1}+1\right) \gamma}-1}{e^{\left(k_{1}+1-s_{1}\right) \gamma}-1}, L(g) \equiv \frac{e^{-\left(k_{1}+1\right) \gamma}-1}{e^{-\left(k_{1}+1-s_{1}\right) \gamma}-1}$, with $1 \leq s_{1} \leq k_{1}$ and $(a, b, c, d)=\frac{k_{1}+1}{k_{1}+1-s_{1}}$

(iii) $L(f) \equiv \frac{e^{s_{1} \gamma}-1}{e^{-\left(k_{1}+1-s_{1}\right) \gamma}-1}, L(g) \equiv \frac{e^{-s_{1} \gamma}-1}{e^{\left.k^{1}+1-s_{1}\right) \gamma}-1}$, with $1 \leq s_{1} \leq k_{1}$ and $(a, b, c, d)=\frac{s_{1}}{s_{1}-k_{1}-1}$.

From Theorem 1.1 we deduce the following theorem, which improves Theorem F.

Theorem 1.5. Let $f$ and $g$ be two distinct nonconstant meromorphic functions sharing $\left(0, k_{1}\right),\left(1, k_{2}\right)$ and $\left(\infty, k_{3}\right)$, where $k_{1}, k_{2}$ and $k_{3}$ are three positive integers satisfying (1.5), and let $a(\neq 0,1)$ be a finite complex number such that (1.3) and (1.13) hold, then $a \notin\left\{-1,2, \frac{1}{2}\right\}$, and there exists a nonconstant entire function $\gamma$ such that $f$ and $g$ are given by one of the three expressions (i), (ii) and (iii) in Theorem $B$.

Remark 1.2. From Example 1.1 and Theorem 1.4 we see that the conditions (1.3) and (1.13) of Theorem 1.5 are necessary.

\section{Some lemmas}

Lemma 2.1([7, Lemma 6]). Let $f$ and $g$ be two distinct nonconstant meromorphic functions such that $f$ and $g$ share 0,1 and $\infty$ IM. If $f$ is a Möbius transformation of $g$, then $f$ and $g$ satisfy one of the following six relations:

(i) $f \cdot g \equiv 1$,

(ii) $(f-1)(g-1) \equiv 1$,

(iii) $f+g \equiv 1$,

(iv) $f \equiv c g$,

(v) $f-1 \equiv c(g-1)$,

(vi) $[(c-1) f+1] \cdot[(c-1) g-c] \equiv-c$, where $c(\neq 0,1)$ is a finite complex number.

Lemma 2.2([2]). Let $f$ and $g$ be two nonconstant meromorphic functions such that $f$ and $g$ share 0,1 and $\infty I M$, then $T(r, f) \leq 3 T(r, g)+S(r, f)$ and $T(r, g) \leq$ $3 T(r, f)+S(r, g)$.

Lemma 2.3 ([14, Lemma 2.6]). Let $f$ and $g$ be two distinct nonconstant meromorphic functions such that $f$ and $g$ share $\left(0, k_{1}\right),\left(1, k_{2}\right)$ and $\left(\infty, k_{3}\right)$, where $k_{1}, k_{2}$ and $k_{3}$ are three positive integers satisfying (1.5). Then

(i) $\bar{N}_{(2}\left(r, \frac{1}{f}\right)+\bar{N}_{(2}\left(r, \frac{1}{f-1}\right)+\bar{N}_{(2}(r, f)=S(r, f)$,

(ii) $\bar{N}_{(2}\left(r, \frac{1}{g}\right)+\bar{N}_{(2}\left(r, \frac{1}{g-1}\right)+\bar{N}_{(2}(r, g)=S(r, f)$.

Lemma 2.4 ([15, Lemma 6$])$. Let $f_{1}$ and $f_{2}$ be two nonconstant meromorphic functions satisfying $\bar{N}\left(r, f_{j}\right)+\bar{N}\left(r, \frac{1}{f_{j}}\right)=S(r)(j=1,2)$. Then either $\bar{N}_{0}\left(r, 1 ; f_{1}, f_{2}\right)=$ $S(r)$ or there exist two integers $s, t(|s|+|t|>0)$ such that $f_{1}^{s} f_{2}^{t} \equiv 1$ where 
$\bar{N}_{0}\left(r, 1 ; f_{1}, f_{2}\right)$ denotes the reduced counting function of $f_{1}$ and $f_{2}$ related to the common 1 - points, and $T(r)=T\left(r, f_{1}\right)+T\left(r, f_{2}\right), S(r)=o(T(r))(r \longrightarrow \infty, r \notin E)$ only depending on $f_{1}$ and $f_{2}$.

Lemma 2.5 ([4, Theorem 1]). Let $f$ and $g$ be two distinct nonconstant meromorphic functions sharing 0,1 and $\infty C M$, and let $a(\neq 0,1)$ be a finite complex number such that (1.3) holds. If $f$ is not any Möbius transformation of $g$, then $f$ and $g$ are given by one of the following three expressions:

(i) $f \equiv \frac{e^{s_{1} \gamma}-1}{e^{\left(k_{1}+1\right) \gamma}-1}, \quad g \equiv \frac{e^{-s_{1} \gamma}-1}{e^{-\left(k_{1}+1\right) \gamma}-1}$, with $1 \leq s_{1} \leq k_{1}$ and $a=\frac{s_{1}}{k_{1}+1}$,

(ii) $f \equiv \frac{e^{\left(k_{1}+1\right) \gamma}-1}{e^{\left(k_{1}+1-s_{1}\right) \gamma}-1}, \quad g \equiv \frac{e^{-\left(k_{1}+1\right) \gamma}-1}{e^{-\left(k_{1}+1-s_{1}\right) \gamma}-1}$, with $1 \leq s_{1} \leq k_{1}$ and $a=\frac{k_{1}+1}{k_{1}+1-s_{1}}$,

(iii) $f \equiv \frac{e^{s_{1} \gamma}-1}{e^{-\left(k_{1}+1-s_{1}\right) \gamma}-1}, \quad g \equiv \frac{e^{-s_{1} \gamma}-1}{e^{\left(k_{1}+1-s_{1}\right)}-1}$, with $1 \leq s_{1} \leq k_{1}$ and $a=\frac{s_{1}}{s_{1}-k_{1}-1}$, where $k_{1}(\geq 2)$ and $s_{1}$ are positive integers such that $s_{1}$ and $k_{1}+1$ are relatively prime, and $\gamma$ is a nonconstant entire function, and such that (1.4) still holds.

Lemma 2.6 ([8, Lemma 2.5] or [15]). Let $s(>0)$ and $t$ be mutually prime integers, and let $c$ be a finite complex number such that $c^{s}=1$, then there exists one and only one common zero of $\omega^{s}-1$ and $\omega^{t}-c$.

Lemma 2.7 ([8, Lemma 2.6] or [9]). Let $f$ be a nonconstant meromorphic function, and let $F=\sum_{k=0}^{p} a_{k} f^{k} / \sum_{j=0}^{q} b_{j} f^{j}$ be an irreducible rational function in $f$ with constant coefficients $\left\{a_{k}\right\}$ and $\left\{b_{j}\right\}$, where $a_{p} \neq 0$ and $b_{q} \neq 0$. Then $T(r, F)=d T(r, f)+O(1)$, where $d=\max \{p, q\}$.

\section{Proof of Theorems}

Proof of Theorem 1.1. We discuss the following two cases.

Case 1. Suppose that $f$ is a Möbius transformation of $g$. Then from (1.3) and Lemma 2.1 we deduce that $f$ and $g$ assume one of the three relations (i), (ii) and (iii) in Lemma 2.1. We discuss the following three subcases.

Subcase 1.1. Suppose that $f$ and $g$ satisfy the relation (i) in Lemma 2.1. Then 0 and $\infty$ are Picard exceptional values of $f$ and $g$. So we let

$$
f=e^{\gamma}
$$

and

$$
g=e^{-\gamma},
$$

where $\gamma$ is a nonconstant entire function. From (3.1) and (3.2) we have

$$
\begin{gathered}
f-a=e^{\gamma}-a, \\
g-a=-\frac{a}{e^{\gamma}} \cdot\left(e^{\gamma}-\frac{1}{a}\right) .
\end{gathered}
$$


Thus from (1.3), (3.3) and (3.4) we deduce (1.6) and

$$
a=\frac{1}{a} .
$$

From (3.5) we get

$$
a=-1 .
$$

From (3.1), (3.2), (3.5) and (3.6) we get (a) of Theorem 1.1.

Subcase 1.2. Suppose that $f$ and $g$ satisfy the relation (ii) in Lemma 2.1. Then 1 and $\infty$ are Picard exceptional values of $f$ and $g$. So we let

$$
f=1+e^{\gamma}
$$

and

$$
g=1+e^{-\gamma},
$$

where $\gamma$ is a nonconstant entire function. From (3.7) and (3.8) and in the same manner as in Subcase 1.1 we get (1.6) and (b) of Theorem 1.1.

Subcase 1.3. Suppose that $f$ and $g$ satisfy the relation (iii) in Lemma 2.1. Then 0 and 1 are Picard exceptional values of $f$ and $g$. So we let

$$
f=\frac{1}{1+e^{\gamma}}
$$

and

$$
g=\frac{1}{1+e^{-\gamma}} .
$$

From (3.9) and (3.10) and in the same manner as in Subcase 1.1 we get (1.6) and (c) of Theorem 1.1.

Case 2. Suppose that $f$ is not any Möbius transformation of $g$. First, from Lemma 2.2 we get

$$
S(r, f)=S(r, g) .
$$

Let

$$
\frac{f-1}{g-1}=h_{1}, \quad \frac{f}{g}=h_{2}
$$

and

$$
h_{0}=\frac{h_{1}}{h_{2}}
$$


then from (3.11)-(3.13) and the above supposition we deduce that none of $h_{0}, h_{1}$ and $h_{2}$ is a constant, and deduce that

$$
f=\frac{h_{1}-1}{h_{0}-1}
$$

and

$$
g=\frac{h_{1}^{-1}-1}{h_{0}^{-1}-1} .
$$

On the other hand, from (3.11) and Lemma 2.3 we deduce

$$
\bar{N}\left(r, \frac{1}{h_{j}}\right)+\bar{N}\left(r, h_{j}\right)=S(r, f)(j=1,2) .
$$

Again from (3.14) and (3.15) we deduce

$$
f-g=\frac{\left(h_{1}-1\right)\left(1-h_{2}^{-1}\right)}{h_{1} h_{2}^{-1}-1} .
$$

From (3.11)-(3.17) we deduce

$$
N_{0}(r)=\bar{N}_{0}\left(r, 1 ; h_{1}, h_{0}\right)+S(r, f)=\bar{N}_{0}\left(r, 1 ; h_{1}, h_{2}\right)+S(r, f) .
$$

Again from (1.3) we deduce

$$
N_{0}(r) \neq S(r, f) .
$$

From (3.18) and (3.19) we get

$$
\bar{N}_{0}\left(r, 1 ; h_{1}, h_{2}\right) \neq S(r, f) .
$$

By (3.16), (3.20) and Lemma 2.4 we know that there exist two integers $s$ and $t$ $(|s|+|t|>0)$ such that

$$
h_{1}^{s} h_{2}^{t} \equiv 1
$$

Substituting (3.12) into (3.21) we get

$$
f^{t}(f-1)^{s} \equiv g^{t}(g-1)^{s} .
$$

Noting that $f$ is not any Möbius transformation of $g$, from (3.22) we deduce that $|s| \cdot|t| \neq 0$ and $|s| \neq|t|$, and so it follows that $f$ and $g$ share 0,1 and $\infty$ CM. Combining Lemma 2.5 and Lemma 2.6 we deduce (1.4) and (i), (ii) and (iii) of Theorem B. Theorem 1.1 is thus completely proved.

Proof of Theorem 1.2. Suppose that $f \not \equiv g$. We discuss the following two cases. 
Case 1. Suppose that $f$ is a Möbius transformation of $g$. Then $f$ and $g$ assume one of the relations (a), (b) and (c) in Theorem 1.1, say, $f$ and $g$ assume the relation (a) in Theorem 1.1, then $a_{1}=a_{2}=-1$, this is impossible.

Case 2. Suppose that $f$ is not any Möbius transformation of $g$. Then $f$ and $g$ assume one of the relations (i), (ii) and (iii) in Theorem B, say, $f$ and $g$ assume the relation (i) in Theorem $\mathrm{B}$, then $a_{1}=a_{2}=\frac{s_{1}}{k_{1}+1}$, this is impossible.

Theorem 1.2 is thus completely proved.

Proof of Theorem 1.3. The assumptions of Theorem 1.3 imply that the conclusion of Theorem 1.1 is valid. We discuss the following two cases.

Case 1. Suppose that $f$ is a Möbius transformation of $g$. Then $f$ and $g$ assume one of the relations (a), (b) and (c) in Theorem 1.1, say, $f$ and $g$ assume the relation (c) in Theorem 1.1, then $a=1 / 2$ and

$$
f-\frac{1}{2}=-\frac{1}{2\left(e^{\gamma}+1\right)} \cdot\left(e^{\gamma}-1\right)
$$

and

$$
f-\frac{1}{2}=-\frac{1}{2\left(e^{-\gamma}+1\right)} \cdot\left(e^{-\gamma}-1\right) .
$$

From (3.23) and (3.24) we deduce (1.9), (1.10) and

$$
\bar{N}_{0}\left(r, \frac{1}{2}, f, g\right)=T(r, f)+S(r, f) .
$$

From (3.23)-(3.25) we get (1.9)-(1.11).

Case 2. Suppose that $f$ is not any Möbius transformation of $g$. Then from Theorem 1.1 we see that $f$ and $g$ assume one of the relations (i), (ii) and (iii) in Theorem B such that (1.4) holds, and so from (1.4) we get (1.11). We discuss the following three subcases.

Subcase 2.1. Suppose that $f$ and $g$ assume the relation (i) in Theorem B, then

$$
\begin{gathered}
f \equiv \frac{e^{\left(k_{1}+1\right) \gamma}-1}{e^{s_{1} \gamma}-1} \\
g \equiv \frac{e^{-\left(k_{1}+1\right) \gamma}-1}{e^{-s_{1} \gamma}-1}
\end{gathered}
$$

and $a=\frac{s_{1}}{k_{1}+1}$, where $k_{1}(\geq 2)$ and $s_{1}$ are positive integers satisfying $1 \leq s_{1} \leq k_{1}$, such that $s_{1}$ and $k_{1}+1$ are relatively prime, and $\gamma$ is a nonconstant entire function. Thus

$$
f-a=\frac{\left(k_{1}+1\right) e^{s_{1} \gamma}-s_{1} e^{\left(k_{1}+1\right) \gamma}+\left(s_{1}-k_{1}-1\right)}{\left(k_{1}+1\right)\left(e^{\left(k_{1}+1\right) \gamma}-1\right)}
$$


and

$$
g-a=\frac{\left(k_{1}+1\right) e^{-s_{1} \gamma}-s_{1} e^{-\left(k_{1}+1\right) \gamma}+\left(s_{1}-k_{1}-1\right)}{\left(k_{1}+1\right)\left(e^{-\left(k_{1}+1\right) \gamma}-1\right)} .
$$

On the other hand, from Lemma 2.6 we deduce that $\omega=1$ is the only common zero of $P_{1}(\omega)=\omega^{k_{1}+1}-1$ and $P_{2}(\omega)=\omega^{s_{1}}-1$. Similarly, $\omega=1$ is the only common zero of $P_{3}(\omega)=\left(k_{1}+1\right) \omega^{s_{1}}-s_{1} \omega^{k_{1}+1}+\left(s_{1}-k_{1}-1\right)$ and $P_{4}(\omega)=\omega^{k_{1}+1}-1$. Thus from (3.26)-(3.29) and Lemma 2.7 we deduce (1.9) and (1.10).

Subcase 2.2. Suppose that $f$ and $g$ assume the relation (ii) in Theorem B, then

$$
\begin{gathered}
f \equiv \frac{e^{\left(k_{1}+1\right) \gamma}-1}{e^{\left(k_{1}+1-s_{1}\right) \gamma}-1}, \\
g \equiv \frac{e^{-\left(k_{1}+1\right) \gamma}-1}{e^{-\left(k_{1}+1-s_{1}\right) \gamma}-1}
\end{gathered}
$$

and $a=\frac{k_{1}+1}{k_{1}+1-s_{1}}$, where $k_{1}(\geq 2)$ and $s_{1}$ are positive integers satisfying $1 \leq s_{1} \leq k_{1}$, such that $s_{1}$ and $k_{1}+1$ are relatively prime, and $\gamma$ is a nonconstant entire function. Thus

$$
f-a=\frac{\left(k_{1}+1-s_{1}\right) e^{\left(k_{1}+1\right) \gamma}-\left(k_{1}+1\right) e^{\left(k_{1}+1-s_{1}\right) \gamma}+s_{1}}{\left(k_{1}+1-s_{1}\right)\left(e^{\left(k_{1}+1-s_{1}\right) \gamma}-1\right)}
$$

and

$$
g-a=\frac{\left(k_{1}+1-s_{1}\right) e^{-\left(k_{1}+1\right) \gamma}-\left(k_{1}+1\right) e^{-\left(k_{1}+1-s_{1}\right) \gamma}+s_{1}}{\left(k_{1}+1-s_{1}\right)\left(e^{-\left(k_{1}+1-s_{1}\right) \gamma}-1\right)} .
$$

From (3.30)-(3.33) and in the same manner as in Subcase 2.1 we deduce (1.9) and (1.10).

Subcase 2.3. Suppose that $f$ and $g$ assume the relation (iii) in Theorem B, then

$$
\begin{gathered}
f \equiv \frac{e^{s_{1} \gamma}-1}{e^{-\left(k_{1}+1-s_{1}\right) \gamma}-1}, \\
g \equiv \frac{e^{-s_{1} \gamma}-1}{e^{\left(k_{1}+1-s_{1}\right) \gamma}-1}
\end{gathered}
$$

and $a=\frac{s_{1}}{s_{1}-k_{1}-1}$, where $k_{1}(\geq 2)$ and $s_{1}$ are positive integers satisfying $1 \leq s_{1} \leq k_{1}$, such that $s_{1}$ and $k_{1}+1$ are relatively prime, and $\gamma$ is a nonconstant entire function. Thus

$$
f-a=\frac{\left(s_{1}-k_{1}-1\right) e^{s_{1} \gamma}-s_{1} e^{-\left(k_{1}+1-s_{1}\right) \gamma}+\left(k_{1}+1\right)}{\left(s_{1}-k_{1}-1\right)\left(e^{-\left(k_{1}+1-s_{1}\right) \gamma}-1\right)}
$$


and

$$
g-a=\frac{\left(s_{1}-k_{1}-1\right) e^{-s_{1} \gamma}-s_{1} e^{\left(k_{1}+1-s_{1}\right) \gamma}+\left(k_{1}+1\right)}{\left(s_{1}-k_{1}-1\right)\left(e^{\left(k_{1}+1-s_{1}\right) \gamma}-1\right)} .
$$

From (3.34)-(3.37) and in the same manner as in Subcase 2.1 we deduce (1.9) and (1.10). Theorem 1.3 is thus completely proved.

Acknowledgment. The authors want to express their thanks to the anonymous referee for his valuable suggestions and comments.

\section{References}

[1] T. C. Alzahary and H. X. Yi, Weighted sharing three values and uniqueness of meromorphic functions, J. Math. Anal. Appl., 295(2004), 247-257.

[2] G. G. Gundersen, Meromorphic functions that share three or four values, J. London Math. Soc., 20 (1979), 457-566.

[3] W. K. Hayman, Meromorphic Functions, The Clarendon Press, Oxford, 1964.

[4] X. M. Li and H. X. Yi, A note on a unicity theorem of N. Terglane, Complex Variables Theory Appl., 48 (2003), 111-118.

[5] I. Lahiri, Weighted sharing and uniqueness of meromorphic functions, Nagoya Math. J., 161 (2001), 193-206.

[6] I. Lahiri, Weighted sharing of three values and uniqueness of meromorphic functions, Kodai Math. J., 24 (2001), 421-435.

[7] I. Lahiri and A. Sarkar, On a uniqueness theorem of Tohge, Arch. Math., 84(2005), 461-469.

[8] X. M. Li and H. X. Yi, Meromorphic functions sharing three values, J. Math. Soc. Japan, 56 (2004), 147-167.

[9] A. Z. Mokhon'ko, On the Nevanlinna characteristics of some meromorphic functions, in: Theory of functions, functional analysis and their applications, Izd-vo Khar'kovsk. Un-ta, 14 (1971), 83-87.

[10] R. Nevanlinna, Le Thérème de Picard-Borel et La Théorie des Fonctions Méromorphes, Gauthier-Villars, Paris, 1929.

[11] N. Terglane, Identitätssätze in C meromorpher Funktionen als Ergebnis von Werteteilung, Diplomarbeit, RWTH Aachen, 1989.

[12] H. X. Yi and C. C. Yang, Uniqueness Theory of Meromorphic Functions, Science Press, Beijing, 1995.

[13] H. X. Yi and X. M. Li, On a result of Terglane concerning uniqueness of meromorphic functions, Indian J. pure appl. Math. 33(2002), 1785-1798.

[14] H. X. Yi, Meromorphic functions with weighted sharing of three values, Complex Variables Theory Appl., 50 (2005), 923-934.

[15] Q. C. Zhang, Meromorphic functions sharing three values, Indian J. Pure Appl. Math., 30 (1999), 667-682. 